\title{
Higher-Order Convergent Iterative Method for Computing the Generalized Inverse over Banach Spaces
}

\author{
Xiaoji Liu and Fu Huang \\ College of Science, Guangxi University for Nationalities, Nanning 530006, China \\ Correspondence should be addressed to Xiaoji Liu; xiaojiliu72@126.com
}

Received 16 May 2013; Revised 24 September 2013; Accepted 24 September 2013

Academic Editor: Qing-Wen Wang

Copyright (C) 2013 X. Liu and F. Huang. This is an open access article distributed under the Creative Commons Attribution License, which permits unrestricted use, distribution, and reproduction in any medium, provided the original work is properly cited.

\begin{abstract}
A higher-order convergent iterative method is provided for calculating the generalized inverse over Banach spaces. We also use this iterative method for computing the generalized Drazin inverse $a^{d}$ in Banach algebra. Moreover, we estimate the error bounds of the iterative methods for approximating $A_{T, S}^{(2)}$ or $a^{d}$.
\end{abstract}

\section{Introduction}

It is well known that the outer generalized inverse has been widely used in various fields, for instance, in statistics, control theory, power systems, nonlinear equations, optimization and numerical analysis, and so on (see [1-15]). Recently, in [16], the authors discussed the iteration (1) for computing $A_{T, S}^{(2)}$ of a given matrix.

Throughout this paper, let $X$ and $Y$ be arbitrary Banach spaces. Then, the symbol $\mathscr{B}(\mathscr{X}, \mathscr{Y})$ denotes the set of all bounded linear operators from $\mathscr{X}$ to $\mathscr{Y}$, in particular, $\mathscr{B}(\mathscr{X}):=\mathscr{B}(\mathscr{X}, \mathscr{X})$. For any $A \in \mathscr{B}(\mathscr{X}, \mathscr{Y})$, we denote its range, null space, and norm by $\mathscr{R}(A), \mathscr{N}(A)$, and $\|A\|$, respectively. Further, we say that $A$ is regular if there exists an $X \in \mathscr{B}(\mathcal{Y}, \mathscr{X})$ such that $A X A=A$ and that $A$ has a $\{2\}$ (or outer) inverse if there exists an $X \in \mathscr{B}(\mathscr{Y}, \mathscr{X})$ such that $X A X=X$. If $A \in \mathscr{B}(\mathscr{X})$, then we denote its spectrum and spectral radius by $\sigma(A)$ and $\rho(A)$, respectively. Let the symbol $L \subset \mathscr{X}$ denote that $L$ is a subspace of $\mathscr{X}$. If $A \in \mathscr{B}(\mathscr{X}, \mathscr{Y})$ and $L \subset \mathscr{X}$, then the restriction $\left.A\right|_{L}$ of $A$ on $L$ is defined by $x \mapsto A x, x \in L$. Let $L, M \subset \mathscr{X}$ with $L \oplus M=\mathscr{X}$. Then, the symbol $P_{L, M}$ stands for an operator that is called a projection from $\mathscr{X}$ onto $L$ if it is a bounded linear map from $\mathscr{X}$ onto $L$ and $P_{L, M}^{2}=P_{L, M}$. It is well known that a closed subspace $L$ of a Banach space $\mathscr{X}$ is complemented in $\mathscr{X}$ if and only if there exists a projection from $\mathscr{X}$ onto $L$.
Let $A \in \mathscr{B}(\mathscr{X}, \mathscr{Y})$ be close; there exists a unique operator $X \in \mathscr{B}(\mathscr{Y}, \mathscr{X})$ such that

$$
\begin{aligned}
\text { (1) } A X A=A & \text { (2) } X A X=X \\
\text { (3) }(A X)^{*}=A X & \text { (4) }(X A)^{*}=X A .
\end{aligned}
$$

Then, $X$ is called the Moore-Penrose inverse of $A$, denoted by $X=A^{\dagger}$. It is well known that $A$ is regular $\Leftrightarrow R(A)$ is closed $\Leftrightarrow A^{\dagger}$ exists.

Throughout this paper, let $\mathscr{A}$ be a complex Banach algebra with the unit 1 . The symbols $a n n^{l}(a)$ and $a n n^{r}(a)$, respectively, stand for the left and right annihilators of $a$ in $\mathscr{A}$. Let $p \in \mathscr{A}$ be idempotent. Then, $p \mathscr{A} p=\{p a p: a \in \mathscr{A}\}$ is a subalgebra of $\mathscr{A}$ with unit $p$. Thus, for $a \in \mathscr{A}$, if there exists an element $b \in p \mathscr{A} p$ such that $a b=b a=p$, then we say that $a$ is invertible in $p \mathscr{A} p$, and $b$ is denoted by $\left.a\right|_{p \mathscr{A} p} ^{-1}$. Recall that an element $b \in \mathscr{A}$ is the generalized Drazin inverse of $a$ (or Koliha-Drazin inverse of $a$ ), if the following hold:

$$
\begin{gathered}
b a b=b, \quad b a=a b, \\
a(1-a b) \text { is quasinilpotent. }
\end{gathered}
$$

If the generalized Drazin inverse of $a$ exists, then it is denoted by $a^{d}$ (see [15] for more details). In particular, if $b=a^{d}$ and 
$a(1-a b)=0$, then $b$ is called the group inverse of $a$ and is denoted by $a_{g}$.

In [17], W. G. Li and Z. Li defined the iterative formula

$$
\begin{array}{r}
X_{k+1} \\
=X_{k}\left[k I-\frac{k(k-1)}{2} A X_{k}+\cdots+(-1)^{k-1}\left(A X_{k}\right)^{k-1}\right], \\
k=2,3, \ldots
\end{array}
$$

In [18], Chen and Wang extended the iterative method (3) proposed by W. G. Li and Z. Li to compute the Moore-Penrose inverse of a matrix. In [19], Liu et al provided the higherorder convergent iterative method (3) in order to calculate the generalized inverse $A_{T, S}^{(2)}$ of a given matrix. In this paper, we will extend the iterative method proposed by W. G. Li and $\mathrm{Z}$. Li in [17] to compute the $\{2\}$-inverse, generalized inverse $A_{T, S}^{(2)}$ over Banach space and also consider the iterative scheme for computing the generalized Drazin inverse $a^{d}$ in Banach algebra.

The paper is organized as follows. Some lemmas will be presented in the remainder of this section. In Section 2, we consider iterative scheme of [19] to compute the generalized inverses $A_{T, S}^{(2)}$ in Banach space. In Section 3, we present iterative formulas for computing the generalized Drazin inverse $a^{d}$ of Banach algebra element $a$.

The following lemmas are needed in what follows.

Lemma 1 (see $[14$, Chapter 1$])$. Let $a \in \mathscr{A}$. Then

(i) $\sigma(a)$ is a nonempty closed subset of $\mathbb{C}$.

(ii) (Spectral mapping theorem for polynomials) if $f$ is a polynomial, then

$$
\sigma(f(a))=f(\sigma(a))
$$

(iii) $\lim _{n \rightarrow 0} a^{n}=0$ if and only if $\rho(a)<1$.

Lemma 2 (see [15, Section 4]). Let $X$ and $Y$ be Banach spaces, and let $A \in \mathscr{B}(\mathscr{X}, \mathcal{Y}), T$ and $S$, respectively, be closed subspaces of $X$ and $Y$. Then, the following statements are equivalent.

(i) A has a $\{2\}$-inverse $B \in B(Y, X)$ such that $\mathscr{R}(B)=T$ and $\mathcal{N}(B)=S$.

(ii) $T$ is a complemented subspace of $X, A(T)$ is closed, $\left.A\right|_{T}: T \rightarrow A(T)$ is invertible, and $A(T) \oplus S=Y$.

In the case when ( $i$ ) or (ii) holds, B is unique and is denoted by $A_{T, S}^{(2)}$.

Lemma 3. Suppose that the conditions of Lemma 2 are satisfied. Then, $A A_{T, S}^{(2)}=P_{A(T), S}$ and $A_{T, S}^{(2)} A=P_{T, T_{1}}$ where $T_{1}=$ $\mathcal{N}\left(A_{T, S}^{(2)} A\right)$. Moreover, for any $G \in \mathscr{B}(\mathcal{Y}, \mathscr{X}), P_{T, T_{1}} G=G \Leftrightarrow$ $\mathscr{R}(G) \subset T ; G P_{A(T), S}=G \Leftrightarrow \mathcal{N}(G) \supset S$.

\section{Higher-Order Convergent Iterative Method for Computing the Generalized Inverse over Banach Spaces}

In this section, we will consider higher-order convergent iterative method for computing the generalized inverse $A_{T, S}^{(2)}$ over Banach spaces. First, we deduce convergent conditions and error bounds of our iterative methods.

Theorem 4. Let $A \in \mathscr{B}(\mathscr{X}, \mathcal{Y}), Y \in \mathscr{B}(\mathscr{Y}, \mathscr{X})$, and let $T \subset \mathscr{X}$ and $S \subset \mathcal{Y}$ both be complemented subspaces, respectively, with $\mathscr{R}(Y)=T, \mathcal{N}(Y)=S$. Define the sequence $\left\{X_{k}\right\}$ in $\mathscr{B}(\mathscr{Y}, \mathscr{X})$ in the following way:

$$
\begin{gathered}
X_{0}=\alpha Y \\
X_{k}=\left[C_{t}^{1} I-C_{t}^{2} X_{k-1} A+\cdots+(-1)^{t-1} C_{t}^{t}\left(X_{k-1} A\right)^{t-1}\right] X_{k-1}
\end{gathered}
$$

it converges to $X_{\infty}$ and $X_{\infty} \in A\{2\}$ with $R\left(X_{\infty}\right)=T$ if and only if $\rho(\alpha Y A-P)<1$ for some scalar $\alpha \in \mathbb{C} \backslash\{0\}$, where $t \geq 2$ is an arbitrary positive integer, $X_{\infty}=\lim X_{k}$, and $P$ is projection from $\mathscr{X}$ onto $T$. Moreover,

(i) if $\mathcal{N}\left(X_{\infty}\right)=S$, then $A_{T, S}^{(2)}$ exists if and only if $\rho(\alpha Y A-$ $P)<1$ for $\alpha \in \mathbb{C} \backslash\{0\}$;

(ii) if $\mathscr{N}\left(X_{\infty}\right)=S$, then $A_{T, S}^{(2)}$ exists.

In particular, if $A_{T, S}^{(2)}$ exists, $\lim X_{k}=A_{T, S}^{(2)}$ and $q=\| \alpha Y A-$ $P \|$, then

$$
\frac{\left\|A_{T, S}^{(2)}-X_{k}\right\|}{\left\|A_{T, S}^{(2)}\right\|} \leq q^{t^{k}}, \quad k \geq 0 .
$$

Proof. From (5), we obtain

$$
\begin{aligned}
{\left[C_{t}^{1} I\right.} & \left.-C_{t}^{2} X_{k-1} A+\cdots+(-1)^{t-1} C_{t}^{t}\left(X_{k-1} A\right)^{t-1}\right] X_{k-1} \\
& =X_{k-1}\left[C_{t}^{1} I-C_{t}^{2} A X_{k-1}+\cdots+(-1)^{t-1} C_{t}^{t}\left(A X_{k-1}\right)^{t-1}\right] .
\end{aligned}
$$

Note that $\mathscr{R}\left(X_{k}\right) \subset \mathscr{R}\left(X_{k-1}\right), k \geq 1$ from (7). Similarly, it is easy to prove that $N\left(X_{k}\right) \supseteq N\left(X_{k-1}\right), k \geq 1$.

Since $\mathscr{R}\left(X_{0}\right)=\mathscr{R}(\alpha Y)=T$ and $\mathscr{N}\left(X_{0}\right)=\mathscr{N}(\alpha Y)=S$, then

$$
\mathscr{R}\left(X_{k}\right) \subset T, \quad \mathcal{N}\left(X_{k}\right) \supset S,
$$

for $k \geq 0$.

From (5), we have

$$
\begin{aligned}
X_{k} A-I & =(-1)^{t+1}\left(X_{k-1} A-I\right)^{t} \\
& =(-1)^{t+1}\left(X_{0} A-I\right)^{t^{k}} .
\end{aligned}
$$

By (8), we get $P X_{k}=X_{k}$. Premultiplying (9) by $P$, then (9) yields

$$
X_{k} A-P=(-1)^{t+1}\left(X_{0} A-P\right)^{t^{k}}
$$


Next, we will investigate the necessary and sufficient condition for the convergent property of the iterative scheme (5). Assume that $\lim X_{k}$ exists; denote by $X_{\infty} \in A\{2\}$ and $\mathscr{R}\left(X_{\infty} A\right)=T$. Then, $\mathscr{R}\left(X_{\infty}\right)=\mathscr{R}\left(X_{\infty} A X_{\infty}\right) \subset \mathscr{R}\left(X_{\infty} A\right) \subset$ $\mathscr{R}\left(X_{\infty}\right)$. Thus, $\mathscr{R}\left(X_{\infty} A\right)=T$ and $\mathscr{X}=T \oplus N\left(X_{\infty} A\right)$; we obtain $X_{\infty} A=P_{T, N\left(X_{\infty} A\right)}$, a projection from $\mathscr{X}$ onto $T$, and $X_{\infty} A X_{k}=X_{k}$ by $(8)$.

Since $P_{T, N\left(X_{\infty} A\right)} X_{0}=X_{0}$, and $X_{k} A-P_{T, N\left(X_{\infty} A\right)}=$ $(-1)^{t+1}\left(X_{0} A-P_{T, N\left(X_{\infty} A\right)}\right)^{t^{k}}$ by (10). Thus,

$$
\begin{aligned}
0 & =\lim X_{k} A-X_{\infty} A=\lim X_{k} A-P_{T, N\left(X_{\infty} A\right)} \\
& =\lim (-1)^{t-1}\left(X_{0} A-P_{T, N\left(X_{\infty} A\right)}\right)^{t^{k}},
\end{aligned}
$$

and then $\rho\left(\alpha Y A-P_{T, N\left(X_{\infty} A\right)}\right)<1$.

Conversely, suppose that $\rho(\alpha Y A-P)<1$ for some scalar $\alpha \in \mathbb{C} \backslash\{0\}$, where $P$ denotes a projection from $\mathscr{X}$ to $T$ and $X$ is complement. Then, $\lim X_{k} A=P$ by (10), and therefore $\lim _{k \rightarrow \infty} X_{k}=\left(\left.A\right|_{T}\right)^{-1}$ and $T=\mathscr{R}(P) \subset \mathscr{R}\left(\lim X_{k}\right)$.

By (8), $R\left(\lim X_{k}\right) \subset T$ because $T$ is close, and then $\mathscr{R}\left(X_{\infty}\right)=T$. Hence, we obtain $\lim X_{k} A \lim X_{k}=\lim X_{k}$. Thus, $\lim X_{k} \in A\{2\}$. It is easy to know that if $N\left(\lim X_{k}\right)=S$, then $\lim X_{k}=A_{T, S}^{(2)}$. Thus, $A_{T, S}^{(2)}$ exists.

Assume that $A_{T S}^{(2)}$ exists. By (8), $N\left(\lim X_{k}\right) \supset S$ because $S$ is closed complement. If $y \in N\left(\lim X_{k}\right) \cup A T$, then $y=A z$ for some $z \in T$. Thus, $0=\lim X_{k} y=\lim X_{k} A z=P z=$ $z$. Thus, $y=0$. Therefore, $N\left(\lim X_{k}\right) \cup A T=\{0\}$ and then $N\left(\lim X_{k}\right)=S$ by Lemma 2. Consequently, $\lim X_{k}=A_{T, S}^{(2)}$.

Since $N\left(X_{k}\right)=S, X_{k} A A_{T, S}^{(2)}=X_{k}$. Thus, postmultiplying (10) by $A_{T, S}^{(2)}$ yields

$$
X_{k}-A_{T, S}^{(2)}=(-1)^{t+1}(\alpha Y A-P)^{t^{k}} A_{T, S}^{(2)} .
$$

Since $A_{T, S}^{(2)}=P A_{T, S}^{(2)}$, we have

$$
\begin{aligned}
\left\|A_{T, S}^{(2)}-X_{k}\right\| & =\left\|(\alpha Y A-P)^{t^{k}} A_{T, S}^{(2)}\right\| \\
& \leq\|\alpha Y A-P\|^{t^{k}}\left\|A_{T, S}^{(2)}\right\| \\
& =q^{t^{k}}\left\|A_{T, S}^{(2)}\right\| .
\end{aligned}
$$

Hence, we get (6).

Similarly, we have the dual result as below.

Theorem 5. Let $A \in \mathscr{B}(\mathscr{X}, \mathscr{Y}), Y \in \mathscr{B}(\mathscr{Y}, \mathscr{X})$, and let $\mathrm{T} \subset$ $\mathscr{X}$ and $S \subset \mathcal{Y}$ both be closed, respectively, with $\mathscr{R}(Y)=T$, $\mathscr{N}(Y)=S$. Define the sequence $\left\{X_{k}\right\} \in \mathscr{B}(\mathscr{Y}, \mathscr{X})$ such that

$$
\begin{gathered}
X_{0}=\alpha Y, \\
X_{k}=X_{k-1}\left[C_{t}^{1} I-C_{t}^{2} A X_{k-1}+\cdots+(-1)^{t-1} C_{t}^{t}\left(A X_{k-1}\right)^{t-1}\right] ;
\end{gathered}
$$

it converges to $X_{\infty}$ and $X_{\infty} \in A\{2\}$ with $\mathcal{N}\left(X_{\infty}\right)=S$ if and only if $\rho(\alpha A Y-Q)<1$ for some scalar $\alpha \in \mathbb{C} \backslash\{0\}$, where $t \geq 2$ is an arbitrary positive integer, $X_{\infty}=\lim X_{k}$, and $Q$ is a projection from $\mathcal{Y}$ onto $S$. Moreover,

(i) if $\mathscr{R}(\Psi)=T$, then $A_{T, S}^{(2)}$ exists if and only if $\rho(\alpha A Y-$ Q) $<1$ for $\alpha \in \mathbb{C} \backslash\{0\}$;

(ii) if $\mathscr{R}(\Psi)=T$, then $A_{T, S}^{(2)}$ exists.

In particular, if $A_{T, S}^{(2)}$ exists, $X_{\infty}=A_{T, S}^{(2)}$ and $q=\|\alpha A Y-Q\|$, then $\left\|A_{T, S}^{(2)}-X_{k}\right\| /\left\|A_{T, S}^{(2)}\right\| \leq q^{t^{k}}, k \geq 0 \cdots$.

Remark 6. Now, we consider how to choose a suitable scalar $\alpha \in \mathbb{C} \backslash\{0\}$ for the iterative scheme (5) such that it converges more faster to $A_{T, S}^{(2)}$.

Since $\mathscr{R}(Y A) \subset T$ and for any $\alpha \in \mathbb{C} \backslash\{0\}, \rho(P-\alpha Y A)=$ $\rho\left(P-\left.\alpha(Y A)\right|_{T}\right)=\max |1-\alpha \mu|\left(\left.\mu \in \sigma(Y A)\right|_{T}\right)$. Therefore, $\rho(P-$ $\alpha Y A)<1$ if and only if $0 \notin \sigma\left(\left.(Y A)\right|_{T}\right)$ and $\max _{\mu \in(Y A)\{\{0\}} \mid 1-$ $\alpha \mu \mid<1$. Thus, there exists $\lambda_{0} \in(Y A) \backslash\{0\}$ with $\left|1-\alpha \lambda_{0}\right|=$ $\rho(P-\alpha Y A)$.

Let $\lambda_{0}=\left|\lambda_{0}\right|(\cos \theta+i \sin \theta)$ and $\alpha=|\alpha|(\cos \varphi+i \sin \varphi)$, where $\theta=\arg \left(\lambda_{0}\right), \varphi=\arg (\alpha)$. Then, $\rho(P-\alpha Y A)=\left[\left|\alpha \lambda_{0}\right|^{2}+\right.$ $\left.1-2\left|\alpha \lambda_{0}\right| \cos (\theta+\varphi)\right]^{1 / 2}$. Thus, $\rho(P-\alpha Y A)<1$ if and only if $0<\left|\alpha \lambda_{0}\right|<2 \cos (\theta+\varphi)$ and $0 \notin \sigma\left(\left.(Y A)\right|_{T}\right)$.

Hence, by $0 \notin \sigma\left(\left.(Y A)\right|_{T}\right)$ and $\alpha$ satisfing $0<|\alpha|<$ $2 \cos (\theta+\varphi) / \rho(Y A)$, we have $\rho(P-\alpha Y A)<1$. In practice, once such a $\lambda_{0}$ is determined, $\alpha$ is taken to satisfy $\arg (\alpha)=$ $-\arg \left(\lambda_{0}\right)$ and $0<|\alpha|<2 / \rho(Y A)$. If $\sigma(Y A)$ is a subset of $\mathbb{R}$, then we take $\alpha$ satisfying $0<|\alpha|<2 / \rho(Y A)$ and $\operatorname{sgn} \alpha=$ $\operatorname{sgn} \lambda_{0}$, where $\lambda_{0} \in \sigma(Y A)$, so as to ensure that $\rho(P-\alpha Y A)<1$.

Assume that $0 \notin \sigma\left(\left.(Y A)\right|_{T}\right)$ hold. In the following, we will obtain the best value $\alpha_{\text {opt }}$ such that $\rho(P-\alpha Y A)$ minimizes for achieving good convergence. Unfortunately, it may be rather difficult. If $\sigma(Y A)$ is a subset of $\mathbb{R}$ and $\lambda_{\min }=\min \{\lambda: \lambda \epsilon$ $\left.\left.\sigma(Y A)\right|_{T}\right\}>0$ analogous to [8, Example 4.1], we can have

$$
\alpha_{\mathrm{opt}}=\frac{2}{\lambda_{\min }+\rho(Y A)} .
$$

In practice, because $\rho(Y A)$ is not easily obtained, we often utilize $\|Y A\|$ instead of it in the above inequations and (15) to choose $\alpha$, which is followed from $\rho(Y A) \leq\|Y A\|$.

\section{Higher-Order Convergent Iterative Method for Computing the Generalized Inverse over Banach Algebra}

In the section, we will investigate a higher-order convergent iterative method for computing the generalized Drazin inverse $a^{d}$ over Banach algebra.

Theorem 7. Let $a \in \mathscr{A}, p \in \mathscr{A}$ be idempotents with ap $=p a$, and $y \in \mathscr{A}$ with $(1-p) y=y(1-p)=y$. Define the sequence $\left\{x_{k}\right\}$ in $\mathscr{A}$ such that

$$
\begin{gathered}
x_{0}=\alpha y, \quad \forall x_{0} \in \mathscr{A}, \\
x_{k}=\left[C_{t}^{1}-C_{t}^{2} x_{k-1} a+\cdots+(-1)^{t-1} C_{t}^{t}\left(x_{k-1} a\right)^{t-1}\right] x_{k-1},
\end{gathered}
$$


where $\alpha \in \mathbb{C} \backslash\{0\}$ and $t \geq 2$. Then the iteration (16) converges to $\lim x_{k}$ and $p x_{0}=0$ if and only if $\rho(1-p-\alpha y a)<1$. In this case, assume that $\operatorname{ann}^{l}(y) \cap(1-p) \mathscr{A}(1-p)=\{0\}$. Then

(i) $a^{d}$ exists and the iteration (16) converges to $a^{d}$ if and only if ap is quasinilpotent in $\mathscr{A}$;

(ii) if $q=(1-p-\alpha y a)<1$, then $\left\|a^{d}-x_{k}\right\| \leq q^{t^{k}}\|y\|$. $\|\alpha\| /\|p+\alpha y a\|$.

Proof. (i) By $(1-p) y=y(1-p)=y$ and $x_{0}=\alpha y$, it implies that $(1-p) x_{0}=x_{0}$. By induction on $k$, we have

$$
\begin{aligned}
& (1-p) x_{k} \\
& \quad=(1-p)\left[C_{t}^{1}-C_{t}^{2} x_{k-1} a+\cdots+(-1)^{t-1} C_{t}^{t}\left(x_{k-1} a\right)^{t-1}\right] x_{k-1} \\
& \quad=x_{k} .
\end{aligned}
$$

By (16), we obtain

$$
\begin{aligned}
x_{k} a-1 & =(-1)^{t-1}\left(x_{k-1} a-1\right)^{t} \\
& =(-1)^{(t-1)^{k}}\left(x_{0} a-1\right)^{t^{k}} .
\end{aligned}
$$

From (17) and (18), we get

$$
\begin{aligned}
(1-p)\left(x_{k} a-1\right) & =x_{k} a-(1-p) \\
& =(-1)^{(t-1)^{k}}\left(x_{0} a-(1-p)\right)^{t^{k}} .
\end{aligned}
$$

The right-hand side of the last equality of (19) implies that

$$
0=\lim _{t \rightarrow \infty}(-1)^{t-1}\left(x_{0} a-(1-p)\right)^{t^{k}}
$$

By $(20)$, we easily have $\rho\left(x_{0} a-(1-p)\right)=\rho(1-p-\alpha y a)<1$.

Conversely, assume that $\rho(1-p-\alpha y a)<1$. Since $p a=a p$ and $(1-p) y=y(1-p)=y,(1-p-\alpha y a) \in(1-p) \mathscr{A}(1-p)$, and then $y a$ is invertible in $(1-p) \mathscr{A}(1-p)$. We will show that $a y$ is invertible in $(1-p) \mathscr{A}(1-p)$. Clearly, ay $\in(1-$ $p) \mathscr{A}(1-p)$, if $a y c=0$ for some $c \in(1-p) \mathscr{A}(1-p)$, then $y c=[(y a)]_{(1-p) \mathscr{A}}^{-1} y a c$. Hence, $c \in \operatorname{ann}^{r}(y) \cap(1-p) \mathscr{A}(1-p)=$ $\{0\}$ and $c=0$. Hence, $0 \notin[(a y)]_{(1-p) \mathscr{A}(1-p)}$, and then $a y$ is invertible in $(1-p) \mathscr{A}(1-p)$.

(i) In the following, we will consider the result (i). It is similar to the deduction of (10), we can write (16) as

$$
x_{k} a=(1-p)+(-1)^{t-1}\left(x_{0} a-(1-p)\right)^{t^{k}}
$$

Thus, postmultiplying (21) by $y$ yields to

$$
x_{k} a y=(1-p) y+(-1)^{t-1}\left(x_{0} a-(1-p)\right)^{t^{k}} y
$$

By Lemma 1 and (22), we prove that $x_{k}$ converges to $y[(a y)]_{(1-p) \mathscr{A}(1-p)}^{-1}$ and is denoted by $x_{\infty}=y[(a y)]_{(1-p) \mathscr{A}(1-p)}^{-1}$. Therefore, $y[(a y)]^{-1}=[(y a)]^{-1} y$ in $(1-p) \mathscr{A}(1-p)$; then

$$
\begin{aligned}
x_{\infty} a & =y[(a y)]_{(1-p) \mathscr{A}(1-p)}^{-1} a \\
& =(y a)_{(1-p) \mathscr{A}(1-p)}^{-1} y a \\
& =1-p=a y[(a y)]_{(1-p) \mathscr{A}(1-p)}^{-1} \\
& =a x_{\infty} .
\end{aligned}
$$

Thus, we obtain $a-a^{2} x_{\infty}=a p$. Since $x_{\infty} a x_{\infty}=$ $\operatorname{ay}[(a y)]_{(1-p) \mathscr{A}(1-p)}^{-1} x_{\infty}=x_{\infty}$, we have that $x_{\infty}=a^{d}$ if and only if ap is quasinilpotent in $\mathscr{A}$.

(ii) Since $p$ is idempotent and $a p=p a$, and

$$
\begin{gathered}
(1-p) y=y(1-p)=y, \\
p(p+\alpha a y) a y=p(p+\alpha y a),
\end{gathered}
$$

then

$$
\begin{aligned}
\alpha(p+\alpha a y)^{-1} a y & =1-(p+\alpha a y)^{-1} p=1-p \\
& =1-p(p+\alpha a y) \\
& =\alpha a y(p+\alpha a y)^{-1} .
\end{aligned}
$$

Therefore, we obtain $(a y)^{-1}=\alpha(p+\alpha a y)^{-1}$ in $(1-p) \mathscr{A}(1-p)$. By (10), we have

$$
x_{k} a y=\left[(1-p)+(-1)^{t+1}[\alpha a y-(1-p)]^{t^{k}}\right] y .
$$

Hence, by the argument in (i) and (26), we have

$$
\begin{aligned}
a^{d}-x_{k}= & x_{\infty}-x_{k} \\
= & y[(a y)]_{(1-p) \mathscr{A}(1-p)}^{-1} \\
& -\left[(1-p)+(-1)^{t+1}[\alpha a y-(1-p)]^{t^{k}}\right] y \\
& \times[(a y)]_{(1-p) \mathscr{A}(1-p)}^{-1} \\
= & (-1)^{t+2}[\alpha a y-(1-p)]^{t^{k}} y \\
& \times[(a y)]_{(1-p) \mathscr{A}(1-p)^{.}}^{-1}
\end{aligned}
$$

Taking limit in (27), then it reduces to (ii).

Similarly, we have the following.

Theorem 8. Let $a \in \mathscr{A}, p \in \mathscr{A}$ be idempotents with ap $=p a$, and $y \in \mathscr{A}$ with $(1-p) y=y(1-p)=y$. Define the sequence $\left\{x_{k}\right\}$ in $\mathscr{A}$ such that

$$
x_{0}=\alpha y, \quad \forall x_{0} \in \mathscr{A} \text {, }
$$

$$
x_{k}=x_{k-1}\left[C_{t}^{1}-C_{t}^{2} a x_{k-1}+\cdots+(-1)^{t-1} C_{t}^{t}\left(a x_{k-1}\right)^{t-1}\right] \text {, }
$$


where $\alpha \in \mathbb{C} \backslash\{0\}$ and $t \geq 2$. Then, the iteration (28) converges to $\lim x_{k}$ and $p x_{0}=0$ if and only if $\rho(1-p-a \alpha y)<1$. In this case, assume that $\operatorname{ann}^{l}(y) \cap(1-p) \mathscr{A}(1-p)=\{0\}$. Then

(i) $a^{d}$ exists and the iteration (28) converges to $a^{d}$ if and only if ap is quasinilpotent in $\mathscr{A}$;

(ii) if $q=(1-p-\alpha y a)<1$, then $\left\|a^{d}-x_{k}\right\| \leq q^{t^{k}}\|y\|$. $\|\alpha\| /\|p+\alpha y a\|$.

\section{Acknowledgments}

This work was supported by the National Natural Science Foundation of China (11061005) and the Ministry of Education Science and Grants (HCIC201103) of Guangxi Key Laboratory of Hybrid Computational and IC Design Analysis Open Fund.

\section{References}

[1] Y. Chen and X. Chen, "Representation and approximation of the outer inverse $A_{T, S}^{(2)}$ of a matrix A, Linear Algebra and Its Applications, vol. 308, no. 1-3, pp. 85-107, 2000.

[2] D. S. Djordjević, P. S. Stanimirović, and Y. Wei, “The representation and approximations of outer generalized inverses," Acta Mathematica Hungarica, vol. 104, no. 1-2, pp. 1-26, 2004.

[3] X. J. Liu, C. M. Hu, and Y. M. Yu, "Further results on iterative methods for computing generalized inverses," Journal of Computational and Applied Mathematics, vol. 234, no. 3, pp. 684694, 2010.

[4] X. J. Liu, Y. M. Yu, and C. M. Hu, “The iterative methods for computing the generalized inverse $A_{T, S}^{(2)}$ of the bounded linear operator between Banach spaces," Applied Mathematics and Computation, vol. 214, no. 2, pp. 391-410, 2009.

[5] R. Penrose, "A generalized inverse for matrices," Proceedings of the Cambridge Philological Society, vol. 51, no. 3, pp. 406-413, 1955.

[6] Y. Saad, Iterative Methods for Sparse Linear Systems, Society for Industrial and Applied Mathematics, Philadelphia, Pa, USA, 2nd edition, 2003.

[7] X. Sheng and G. Chen, "Several representations of generalized inverse $A_{T, S}^{(2)}$ and their application," International Journal of Computer Mathematics, vol. 85, no. 9, pp. 1441-1453, 2008.

[8] X. Sheng, G. Chen, and Y. Gong, "The representation and computation of generalized inverse $A_{T, S}^{(2)}$, Journal of Computational and Applied Mathematics, vol. 213, no. 1, pp. 248-257, 2008.

[9] G. Wang, Y. Wei, and S. Qiao, Generalized Inverses: Theory and Computations, Science Press, Beijing, China, 2004.

[10] Y. Wei, "A characterization and representation of the generalized inverse $A_{T S}^{(2)}$ and its applications," Linear Algebra and Its Applications, vol. 280, no. 2-3, pp. 87-96, 1998.

[11] Y. Wei and H. Wu, "The representation and approximation for the generalized inverse $A_{T, S}^{(2)}$," Applied Mathematics and Computation, vol. 135, no. 2-3, pp. 263-276, 2003.

[12] Y. Yu and Y. Wei, "The representation and computational procedures for the generalized inverse $A_{T, S}^{(2)}$ of an operator a in hilbert spaces," Numerical Functional Analysis and Optimization, vol. 30, no. 1-2, pp. 168-182, 2009.

[13] J. J. Koliha, “A generalized drazin inverse," Glasgow Mathematical Journal, vol. 38, no. 3, pp. 367-381, 1996.
[14] V. Müler, Spectral Theory of Linear Operators and Spectral Systems in Banach Algebras, Birkhäuser, Basel, Switzerland, 2nd edition, 2007.

[15] D. S. Djordjević and P. S. Stanimirović, "On the generalized Drazin inverse and generalized resolvent," Czechoslovak Mathematical Journal, vol. 51, no. 3, pp. 617-634, 2001.

[16] A. J. Getson and F. C. Hsuan, 2-Inverses and Their Statistical Application, Springer, New York, NY, USA, 1988.

[17] W. G. Li and Z. Li, "A family of iterative methods for computing the approximate inverse of a square matrix and inner inverse of a non-square matrix," Applied Mathematics and Computation, vol. 215, no. 9, pp. 3433-3442, 2010.

[18] H. Chen and Y. Wang, "A Family of higher-order convergent iterative methods for computing the Moore-Penrose inverse," Applied Mathematics and Computation, vol. 218, no. 8, pp. 40124016, 2011.

[19] X. Liu, H. Jin, and Y. Yu, "Higher-order convergent iterative method for computing the generalized inverse and its application to Toeplitz matrices," Linear Algebra and its Applications, vol. 6, pp. 1635-1650, 2013. 


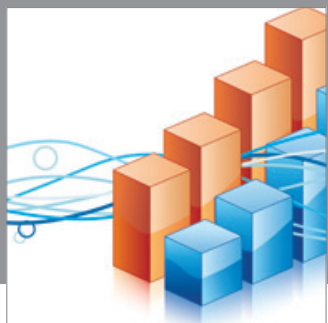

Advances in

Operations Research

mansans

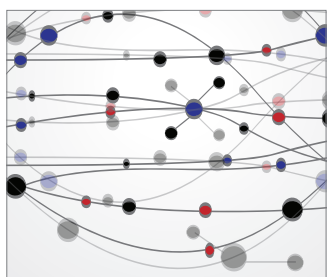

The Scientific World Journal
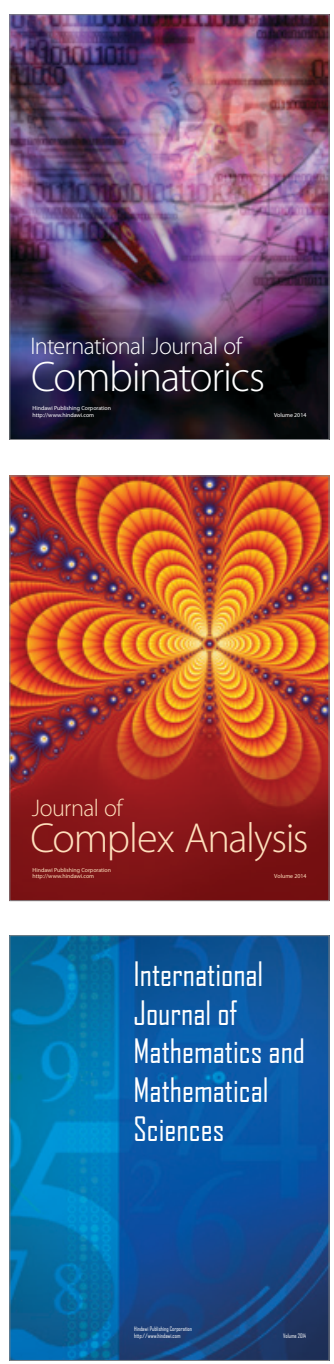
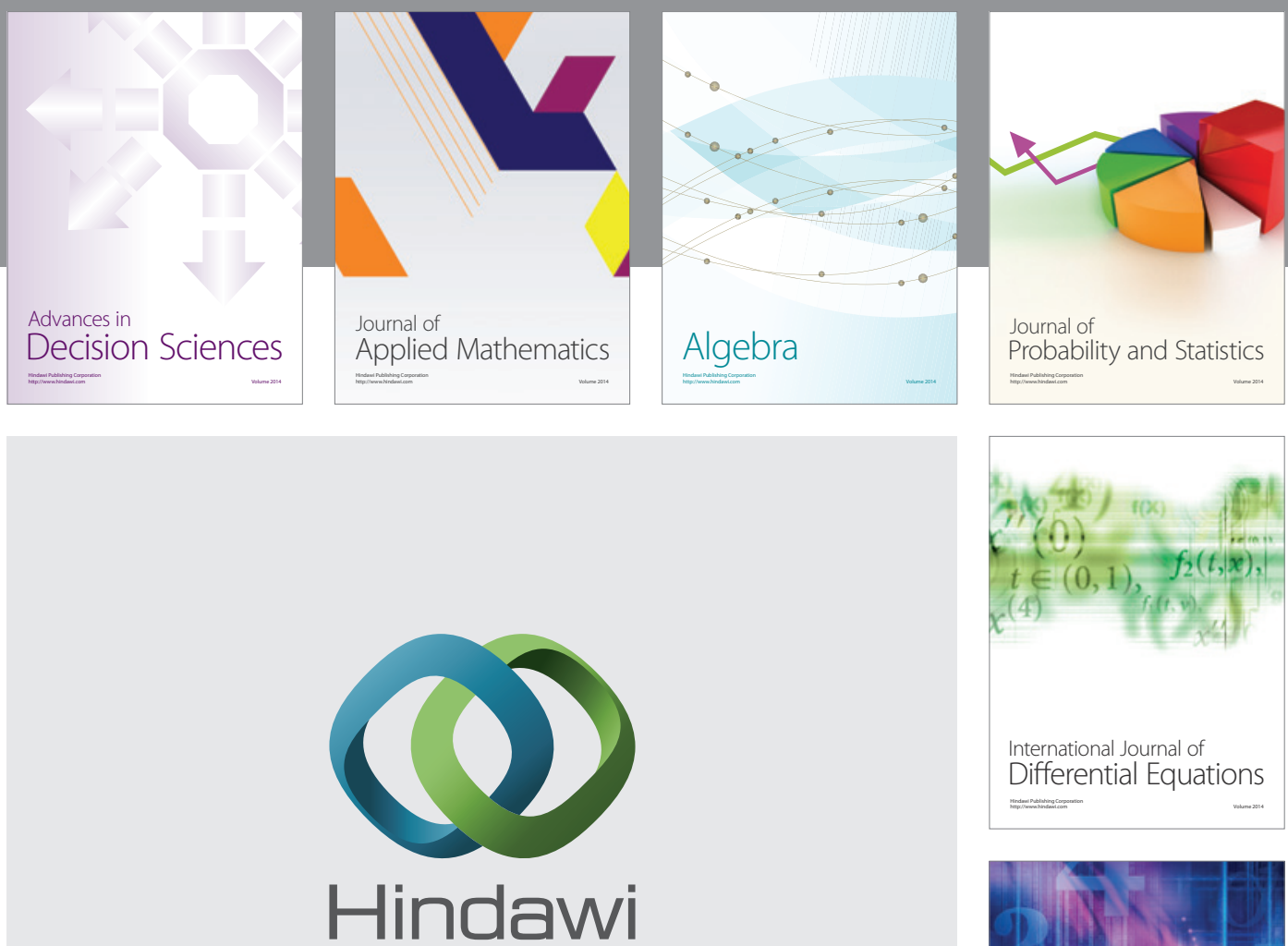

Submit your manuscripts at http://www.hindawi.com
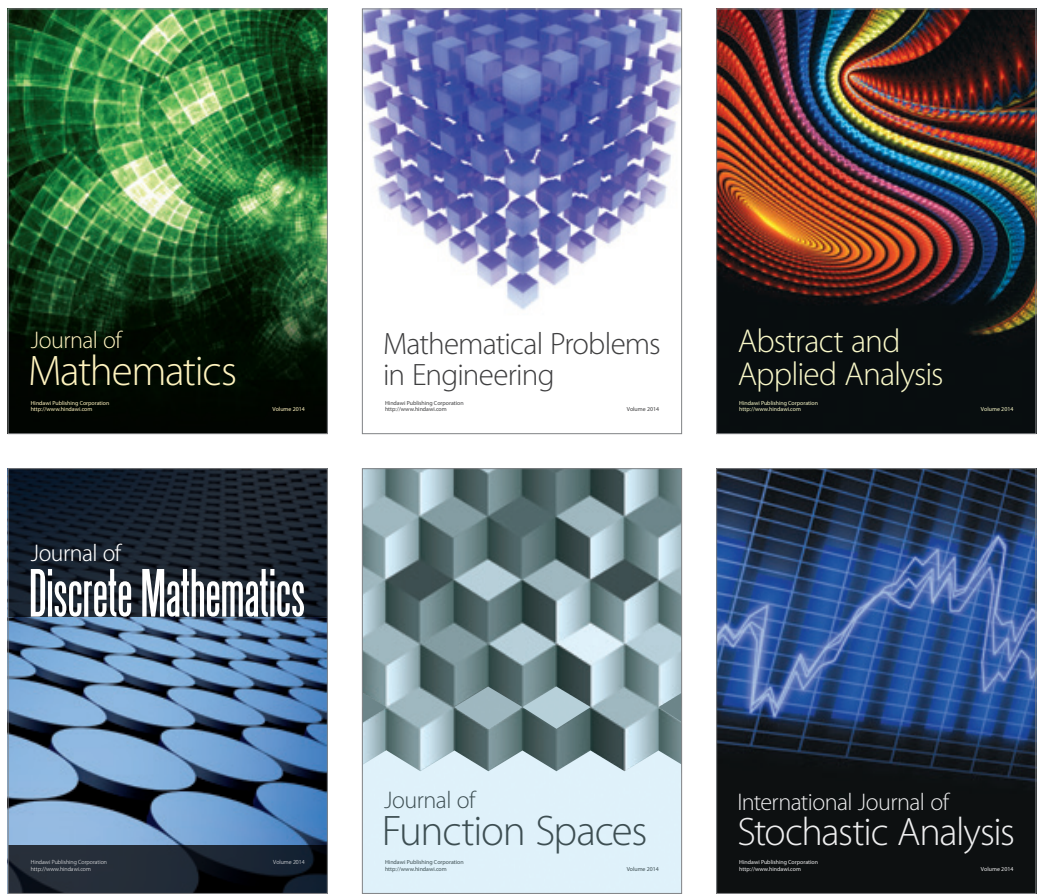

Journal of

Function Spaces

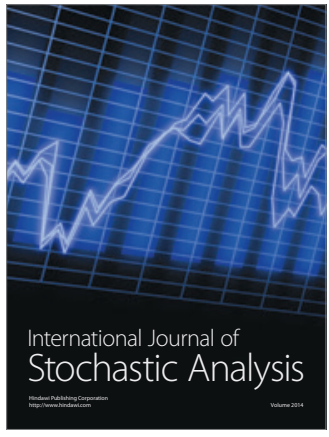

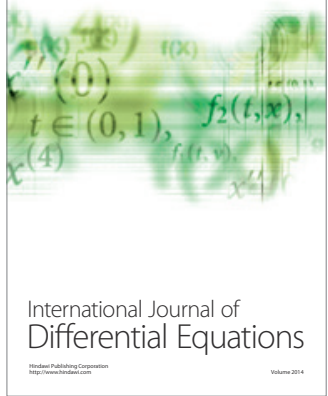
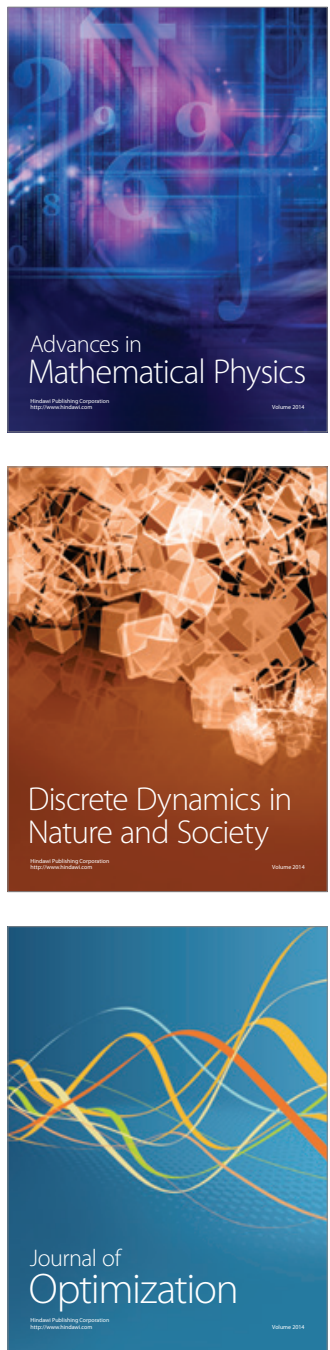